\title{
Regional anesthesia in the time of COVID-19: a minireview
}

\author{
Kunihisa Hotta ${ }^{1}$ (i)
}

Received: 6 July 2020 / Accepted: 18 July 2020 / Published online: 25 July 2020

(C) Japanese Society of Anesthesiologists 2020

\begin{abstract}
The treatment of surgical patients who are confirmed or suspected of coronavirus disease 2019 (COVID-19) is a challenge for all anesthesiologists. The safety of both patients and healthcare workers should be taken into consideration when performing anesthesia management for patients with COVID-19. General anesthesia requiring airway intervention may exacerbate COVID-19 pneumonia, and aerosol generation during airway intervention risks COVID-19 transmission to medical staff. However, regional anesthesia is not an aerosol-generating procedure. The neuraxial anesthesia may have little adverse influence on clinical outcomes in patients with COVID-19 after reviewing previous case reports. Regional anesthesia may have some advantages over general anesthesia for this group of patients, but unplanned conversion to general anesthesia during surgery is not preferred. Thus, careful consideration should be given to ensure that the surgery is performed entirely under regional anesthesia. The use of ultrasound guidance and the performance by an experienced physician may reduce the incidence of failed block and complications. The use of long-acting local anesthetic prolongs the anesthetic effect of regional anesthesia. Besides, a safe and sufficient dose of local anesthetic should be used.
\end{abstract}

Keywords COVID-19 $\cdot$ Regional anesthesia $\cdot$ Neuraxial anesthesia $\cdot$ Peripheral nerve block

\section{Introduction}

Severe acute respiratory syndrome coronavirus 2 (SARSCoV-2) caused the coronavirus disease 2019 (COVID-19) epidemic worldwide, which results in great concern and confusion to our society. Although most patients with COVID19 experience a mild to moderate clinical course, some of the patients develop severe, sometimes fatal, pneumonia. There have been no drugs approved for the treatment of COVID-19 yet. The treatment of surgical patients who are confirmed or suspected of this disease is a challenge for all anesthesiologists. General anesthesia requiring airway intervention may exacerbate COVID-19 pneumonia. Aerosol generation during airway intervention risks COVID-19 transmission to medical staff. However, regional anesthesia may have some advantages over general anesthesia for this group of patients. This minireview focuses on regional

Kunihisa Hotta

hottak@jichi.ac.jp

1 Department of Anesthesiology and Critical Care Medicine, Jichi Medical University School of Medicine, 3311-1 Yakushiji, Shimotsuke-shi, Tochigi 329-0498, Japan anesthesia in patients with COVID-19 and discusses the practical consideration for performing regional anesthesia.

\section{Anesthesia method: general or regional?}

The safety of both patients and healthcare workers should be taken into consideration when performing anesthesia management for patients who are confirmed or suspected to have COVID-19. Patients with acute and recent respiratory infections are at high risk of pulmonary complications during the perioperative period [1]. A history of respiratory infection within a month is reported to be an independent predictor of risk for postoperative pulmonary complications [2]. Thus, nonurgent surgeries in patients with respiratory infections, including COVID-19, should be postponed and rescheduled after infection treatment. In addition, general anesthesia requiring airway intervention has a higher risk of perioperative pulmonary complications than regional anesthesia [3,4]. A previous systematic review reported that the incidence of postoperative pneumonia in patients undergoing neuraxial anesthesia was lower than that in patients undergoing general anesthesia (odds ratio, 0.63) [5]. Thus, 
it is reasonable to prioritize regional anesthesia over general anesthesia to minimize the risks of exacerbating respiratory infection and causing pulmonary complications in patients with COVID-19.

It has not yet been revealed how regional anesthesia adversely affects the health of the patients with COVID-19 because of insufficient clinical experience and clinical trials. Based on a review of previous case reports, the neuraxial anesthesia may have little adverse influence on clinical outcomes in patients with COVID-19 [6-9]. A single-center retrospective observational study assessed the clinical characteristics and outcomes for 49 patients with COVID-19 who underwent cesarean section or orthopedic surgeries under spinal anesthesia [6]. Perioperative hemodynamic status and oxygenation remained stable. No patients developed severe pneumonia or died of pneumonia in the postoperative period. A case series of 17 COVID-19-positive parturients undergoing cesarean section indicated the safety and efficacy of both epidural and general anesthesia [7]. Intraoperative hypotension (systolic blood pressure of $<80 \%$ at baseline) occurred in 12 (86\%) of 14 patients who received continuous epidural anesthesia. The authors noted that no adverse end-organ damage was seen because of the short duration of the hemodynamic change. Another case series of 43 COVID-19-positive pregnant women reported 18 cases of delivery [8]. All deliveries were performed under neuraxial anesthesia, including eight cases of cesarean section. No hemodynamic instability and neurologic complications were noted in any of the patients. Transient hypotension occurred during surgery, which was stabilized by methoxamine, in a case report of a pregnant woman with COVID-19 pneumonia who underwent an emergency cesarean section with spinal anesthesia [9].

The risk of COVID-19 being transmitted to healthcare workers is another important issue when selecting the type of anesthesia. General anesthesia accompanied by airway intervention leads to aerosol generation during bag-mask ventilation, tracheal intubation, suction of the airway tract, and extubation. Particularly during these procedures, anesthesiologists should be careful to avoid exposure to aerosols and droplets. According to a systematic review that analyzed the transmission risk of acute respiratory infections to healthcare workers for aerosol-generating procedures, the odds ratio of tracheal intubation in four cohort and four casecontrol studies have been reported to be 6.6 [10]. However, regional anesthesia is not an aerosol-generating procedure, and the risk of COVID-19 transmission is theoretically lower with regional anesthesia than with general anesthesia. The American Society of Regional Anesthesia and Pain Medicine and European Society of Regional Anesthesia and Pain Therapy have made practice recommendations on neuraxial anesthesia and peripheral nerve blocks during the COVID19 pandemic. https://www.asra.com/page/2905/pract ice-recommendations-on-neuraxial-anesthesia-and-perip heral-nerve-blocks-dur. Accessed 28 June 2020. In their joint statement, regional anesthesia is not contraindicated for COVID-19-positive patients and should be preferred for providing anesthesia care wherever possible. However, proper infection control against droplets and contaminated sources is necessary even when performing regional anesthesia.

When planning regional anesthesia, we have to keep in mind the possibilities of failed block and unplanned conversion to general anesthesia. In such cases, the risk of COVID19 transmission from patient's droplets and aerosols associated with airway intervention will be increased. Limited staff and equipment to prevent the transmission of infection can be a risk to the safety of airway management and the certainty of infection control. Regional anesthesia has some advantages over general anesthesia in patients with COVID19 , but unplanned conversion to general anesthesia during surgery is not preferred. Thus, careful consideration should be given to ensure that the surgery is performed entirely under regional anesthesia. It may be better to start with general anesthesia rather than planning low-probability regional anesthesia. General anesthesia should also be chosen in critically ill patients who are already intubated.

\section{Practical considerations for performing regional anesthesia in patients with COVID-19}

\section{Preprocedural evaluation}

The indication and contraindication to neuraxial anesthesia and peripheral nerve block do not differ in patients with or without COVID-19. In clinical practice, coagulopathy and antithrombotic therapy are common contraindications to regional anesthesia. An early report from China suggested a frequent incidence of thrombocytopenia in patients with severe COVID-19 [11]. The frequency of patients with platelet counts below $150,000 / \mathrm{mm}^{3}$ was higher in patients categorized as severe $(57.7 \%)$ than in those categorized as nonsevere $(31.6 \%)$. A subsequent meta-analysis confirmed this finding [12].

\section{Patients with reduced respiratory reserve}

In patients with reduced respiratory reserve caused by pulmonary infection and coexisting pulmonary disease, blockade of respiratory muscles by regional anesthesia can lead to further impairment. Distal approaches such as axillary approach are less likely to interfere with patient's respiratory function because the brachial plexus block interscalene approach frequently causes phrenic nerve palsy. Phrenic nerve palsy in cases of shoulder surgery 
may be avoided by limiting the dose and injection volume of local anesthetic with the interscalene approach or by performing a combination of selective suprascapular and axillary nerve blocks [13].

\section{Sedation and oxygen administration}

Intraoperative sedatives used with regional anesthesia have some respiratory depressant effect, increasing the need for oxygen administration and airway manipulation or intervention. Therefore, the use of sedation should be minimal or avoided in patients with COVID-19, and respiratory status should be monitored when patients are sedated. Oxygen administration may spread droplets and is reported to be an independent risk factor for nosocomial outbreaks of SARS [14]. The flow of oxygen via face mask and nasal cannula should be kept low enough to maintain percutaneous oxygen saturation. The patient should wear a surgical mask at all times because even normal breathing and speaking are capable of producing aerosols.

\section{Safe and reliable performance of regional anesthesia}

Safe and reliable performance of regional anesthesia is more preferred than usual to avoid unplanned medical intervention such as conversion to general anesthesia, treatment of local anesthetic systemic toxicity, and epidural blood patch for postdural puncture headache. The use of ultrasound guidance and the performance by an experienced physician may reduce the incidence of failed block and complications. The use of long-acting local anesthetic prolongs the anesthetic effect of regional anesthesia, which contributes to avoiding conversion to general anesthesia and reducing postoperative opioids. Besides, a safe and sufficient dose of local anesthetic should be used.

\section{Infection protection}

Although regional anesthesia is considered to have a lower risk of COVID-19 transmission than general anesthesia, safety protocols should be followed to prevent infection from droplets and contaminated sources. Personal protective equipment includes a surgical mask, eye protection, surgical gown, and double glove. The use of N95 masks should be considered depending on the risk of aerosol generation and droplet spread. Restrictions of staff and equipment in the operating room should be considered to minimize exposure to the virus.

\section{References}

1. Tait AR, Malviya S, Voepel-Lewis T, Munro HM, Seiwert M, Pandit UA. Risk factors for perioperative adverse respiratory events in children with upper respiratory tract infections. Anesthesiology. 2001;95:299-306. https://doi.org/10.1097/00000542200108000-00008.

2. Canet J, Gallart L, Gomar C, Paluzie G, Vallès J, Castillo J, Sabaté S, Valentín M, Briones Z, Sanchis H, ARISCAT G. Prediction of postoperative pulmonary complications in a population-based surgical cohort. Anesthesiology. 2010;113:1338-500. https://doi. org/10.1097/ALN.0b013e3181fc6e0a.

3. Warren J, Sundaram K, Anis H, Kamath AF, Mont MA, Higuera CA, Piuzzi NS. Spinal anesthesia is associated with decreased complications after total knee and hip arthroplasty. J Am Acad Orthop Surg. 2020;28:e213-e221221. https://doi.org/10.5435/ JAAOS-D-19-00156.

4. Neuman MD, Silber JH, Elkassabany NM, Ludwig JM, Fleisher LA. Comparative effectiveness of regional versus general anesthesia for hip fracture surgery in adults. Anesthesiology. 2012;117:72-92. https://doi.org/10.1097/ALN.0b013e3182545e7 c.

5. Rodgers A, Walker N, Schug S, McKee A, Kehlet H, van Zundert A, Sage D, Futter M, Saville G, Clark T, MacMahon S. Reduction of postoperative mortality and morbidity with epidural or spinal anaesthesia: results from overview of randomised trials. BMJ. 2000;321:1493. https://doi.org/10.1136/bmj.321.7275.1493.

6. Zhong Q, Liu YY, Luo Q, Zou YF, Jiang HX, Li H, Zhang JJ, Li Z, Yang X, Ma M, Tang LJ, Chen YY, Zheng F, Ke JJ, Zhang ZZ. Spinal anaesthesia for patients with coronavirus disease 2019 and possible transmission rates in anaesthetists: retrospective, singlecentre, observational cohort study. Br J Anaesth. 2020;124:670-5. https://doi.org/10.1016/j.bja.2020.03.007.

7. Chen R, Zhang Y, Huang L, Cheng BH, Xia ZY, Meng QT. Safety and efficacy of different anesthetic regimens for parturients with COVID-19 undergoing Cesarean delivery: a case series of 17 patients. Can J Anesth. 2020;67:655-63. https://doi.org/10.1007/ s12630-020-01630-7.

8. Breslin N, Baptiste C, Gyamfi-Bannerman C, Miller R, Martinez R, Bernstein K, Ring L, Landau R, Purisch S, Friedman AM, Fuchs K, Sutton D, Andrikopoulou M, Rupley D, Sheen JJ, Aubey J, Zork N, Moroz L, Mourad M, Wapner R, Simpson LL, D’Alton ME, Goffman D. Coronavirus disease 2019 infection among asymptomatic and symptomatic pregnant women: two weeks of confirmed presentations to an affiliated pair of New York City hospitals. Am J Obstet Gynecol MFM. 2020;2:100118. https:// doi.org/10.1016/j.ajogmf.2020.100118.

9. Xia H, Zhao S, Wu Z, Luo H, Zhou C, Chen X. Emergency Caesarean delivery in a patient with confirmed COVID-19 under spinal anaesthesia. Br J Anaesth. 2020;124:e216-e218218. https:// doi.org/10.1016/j.bja.2020.02.016.

10. Tran K, Cimon K, Severn M, Pessoa-Silva CL, Conly J. Aerosol generating procedures and risk of transmission of acute respiratory infections to healthcare workers: a systematic review. PLoS ONE. 2012;7(4):e35797.

11. Guan WJ, Ni ZY, Hu Y, Liang WH, Ou CQ, He JX, Liu L, Shan H, Lei CL, Hui DSC, Du B, Li L, Zeng G, Yuen KY, Chen R, Tang C, Wang T, Chen P, Xiang J, Li S, Wang JL, Liang Z, Peng Y, Wei L, Liu Y, Hu YH, Peng P, Wang JM, Liu J, Chen Z, Li G, Zheng ZJ, Qiu S, Luo J, Ye C, Zhu S, Zhong N. China Medical treatment expert group for COVID-19. Clinical characteristics of coronavirus disease 2019 in China. N Engl J Med. 2020;382:1708-20. https://doi.org/10.1056/NEJMoa2002032.

12. Lippi G, Plebani M, Henry BM. Thrombocytopenia is associated with severe coronavirus disease 2019 (COVID-19) infections: 
a meta-analysis. Clin Chim Acta. 2020;506:145-8. https://doi. org/10.1016/j.cca.2020.03.022.

13. El-Boghdadly K, Chin KJ, Chan VWS. Phrenic nerve palsy and regional anesthesia for shoulder surgery: anatomical, physiologic, and clinical considerations. Anesthesiology. 2017;127:173-91. https://doi.org/10.1097/ALN.0000000000001668.

14. Yu IT, Xie ZH, Tsoi KK, Chiu YL, Lok SW, Tang XP, Hui DS, Lee N, Li YM, Huang ZT, Liu T, Wong TW, Zhong NS, Sung JJ. Why did outbreaks of severe acute respiratory syndrome occur in some hospital wards but not in others? Clin Infect Dis. 2007;44:1017-25. https://doi.org/10.1086/512819.

Publisher's Note Springer Nature remains neutral with regard to jurisdictional claims in published maps and institutional affiliations. 